\title{
Searching for Continuous Gravitational Waves with Pulsar Timing Arrays: Detection and Characterization
}

\author{
Jusitn Ellis \\ The Leonard E. Parker Center for Gravitation, Cosmology \& Astrophysics \\ Department of Physics, University of Wisconsin-Milwaukee \\ Milwaukee, WI USA
}

\begin{abstract}
Gravitational Waves (GWs) are tiny ripples in the fabric of space-time predicted by Einsteins theory of General Relativity. Pulsar timing arrays (PTAs) offer a unique opportunity to detect low frequency GWs in the near future. Such a detection would be complementary to both LISA and LIGO GW efforts. In this frequency band, the expected source of GWs are Supermassive Black Hole Binaries (SMBHBs) and they will most likely form in an ensemble creating a stochastic GW background with the possibility of a few nearby/massive sources that will be individually resolvable. A direct detection of GWs will open a new window into the fields of astronomy and astrophysics by allowing us to constrain the coalescence rate of SMBHBs, providing further tests on the theory of General Relativity, and giving us access to properties of black holes not accessible by current astronomical techniques. Here we will discuss the development of a robust detection pipeline for single resolvable GW sources that is fully tailored to the unique aspects of PTA data analysis.
\end{abstract}

\section{Introduction}

In the next few years pulsar timing arrays (PTAs) are expected to detect gravitational waves $(\mathrm{GWs})$ in the frequency range $10^{-9} \mathrm{~Hz}-10^{-7} \mathrm{~Hz}$. Potential sources of GWs in this frequency range include supermassive black hole binary systems (SMBHBs) Sesana et al. (2008), cosmic (super)strings Olmez et al. (2010), inflation Starobinsky (1979), and a first order phase transition at the QCD scale Caprini et al. (2010). The community has thus far mostly focused on stochastic backgrounds produced by these sources, however; sufficiently nearby single SMBHBs may produce detectable continuous waves with periods on the order of years and masses in the range $10^{8} M_{\odot}-10^{9} M_{\odot}$ Wyithe \& Loeb (2003); Sesana et al. (2009); Sesana \& Vecchio (2010). The concept of a PTA, an array of accurately timed millisecond pulsars, was first conceived of over two decades ago Romani (1989); Foster \& Backer (1990). Twenty years later three main PTAs are in full operation: the North American Nanohertz Observatory for Gravitational waves (NANOGrav; Jenet et al. (2009)), the Parkes Pulsar Timing Array (PPTA; Manchester (2008)), and the European Pulsar Timing Array (EPTA; Janssen et al. (2008)). The three PTAs collaborate to form the International Pulsar Timing Array (IPTA; Hobbs et al. (2010)).

Many authors have focused on determining the parameter accuracy that we may hope to extract from a future detection of a continuous GW from a SMBMB. Corbin \& Cornish (2010) have developed a Bayesian Markov Chain Monte-Carlo (MCMC) data analysis algorithm for

\footnotetext{
JE is partially funded by the Wisconsin Space Grant Consortium and the NSF through PIRE award number 0968126.
} 
parameter estimation of a SMBHB system in which the perturbation due to the GW at the pulsar is taken into account in the detection scheme, thereby increasing the signal-to-noiseratio (SNR) and improving the accuracy of the GW source location on the sky. Recently, Lee et al. (2011) have developed parameter estimation techniques incorporating the pulsar term and have placed limits on the minimum detectable amplitude of a continuous GW source.

In this article, we will briefly review detection and characterization techniques developed for analysis of real PTA data (Ellis, 2013).

\section{Methods}

The GW signal from a SMBHB in a circular orbit measured at the earth can be described by 8 parameters: 2 intrinsic to the binary and 5 that are extrinsic and depend on our line of sight to the binary. The intrinsic parameters are the total mass and orbital separation (or equivalently the period of the binary, though Kepler's 3rd law) of the binary system and the extrinsic parameters are the sky location of the binary, initial phase at the time of observation, distance to the binary and orientation of the binary in the sky projected onto our line of sight. Since we are using the pulsars as our GW detector we must know the distance to the pulsars in order to correctly measure the GW parameters. However, typical pulsar distance uncertainties are on the order of tens of percent (Verbiest et al., 2012), in order to attain phase coherence in our search algorithm, we must allow the pulsar distance to vary as a search parameter as well.

Our parameter space will be at least 9-dimensional for a PTA comprised of one pulsar and we will gain another parameter for every pulsar that is used in the search. For typical PTAs (20 pulsars), this means that the parameter space will be $\sim 28$ dimensional. For this reason we have chosen to use a Markov Chain Monte-Carlo (MCMC) algorithm to perform our search and parameter estimation. MCMC is a stochastic sampling method that will efficiently explore large parameter spaces and map out the probability distribution function (pdf) for the model parameters. This is accomplished through the Metropolis-Hastings algorithm that allows the sampler to focus on high probability areas of parameter space while still exploring the entire prior volume. Next we will describe how this algorithm is used to search the parameter space for the maximum likelihood values, map out the pdfs of all parameters and make statements about the detection of GWs in our data set. 


\section{Characterization}

Our goal is to measure both intrinsic and extrinsic parameters of the SMBMB through GW observations. To do this we must explore the large parameter space of the GW parameters in addition to the pulsar distances themselves. To efficiently locate the global maximum in the parameter space we make use of parallel tempering. Parallel tempering involves running several MCMC chains in parallel each evaluating the likelihood function of our data raised to some power $1 / T$, where $T$ ranges from 1 to $T_{\max }$, where $T=1$ represents the true likelihood function and higher temperatures are a flatter version of the true likelihood function. This allows the hotter chains to explore the likelihood surface much more quickly and the algorithm then communicates information from the hotter chains back down to the $T=1$ chain. Further discussion of the setup of parallel tempering is beyond the scope of this document, suffice it to say that this step is critical in locating the maximum likelihood quickly. We have simulated a moderately strong GW source and have run our analysis. Figure 1 shows that the algorithm quickly locates the maximum likelihood and injected parameters.
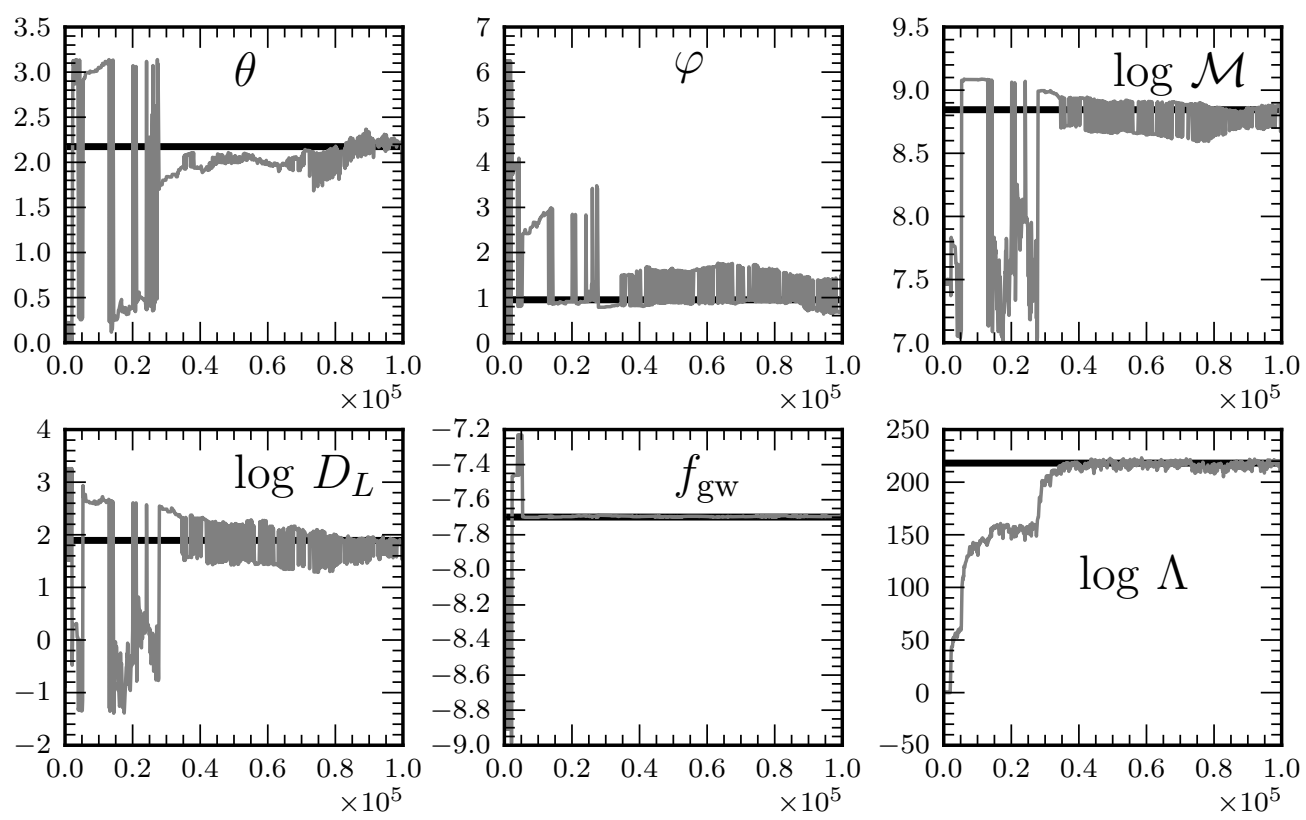

Figure 1: Trace plots for the measurable parameters (the inclination angle, initial phase and polarization angle are not well constrained for this realization) for an $\mathrm{SNR}=20$ injection for the first $10^{5}$ steps. In all cases the green line represents the injected parameters and the blue is the chain trace. We can see that the parallel tempering scheme has allowed us to locate the global maxima of the log-likelihood and all parameters within the first $\sim 6 \times 10^{4}$ steps. 
Once we have located the maximum likelihood we can begin to collect samples of the pdfs of the model parameters. This phase of the algorithm is called the characterization phase. During this phase we will learn about any correlations among parameters or about any multimodal structure in the likelihood surface. As an example, we show the 2-d pdfs of the sky location and mass and distance to the source in Figure 2.
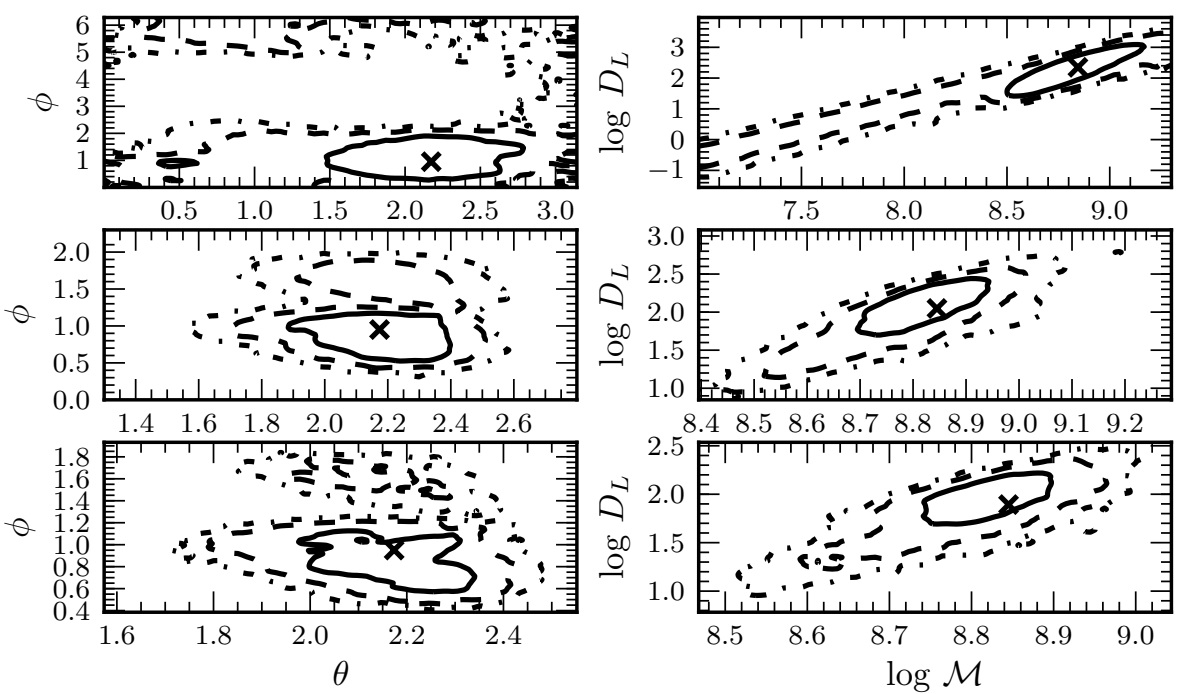

Figure 2: Marginalized 2-D posterior pdfs in the sky coordinates $(\theta, \phi)$ and the log of the chirp mass and distance $\left(\log \mathcal{M}, \log D_{L}\right)$ for injected SNRs of 7, 14, and 20 shown from top to bottom. Here the injected GW source is in the direction of the Fornax cluster with chirp mass $\mathcal{M}=7 \times 10^{8} M_{\odot}$. The distance to the source is varied to achieve the desired SNR. Here the " $\times$ " marker indicates the injected parameters and the solid, dashed and dot-dashed lines represent the 1, 2, and 3 sigma credible regions, respectively.

Here we see that we can recover the sky location with a smaller error box for louder GW signals. In addition, with a louder signal we can break the degeneracy between the mass of the system and distance to the source.

\section{Detection}

Above, we have shown that we are able to characterize the parameters of the SMBHB source if it is loud enough in our data. In this section we will review how we evaluate the evidence for the presence of a GW in our PTA data set. In Bayesian statistics we directly compare the evidence for a model with and without a GW source. In practice computing the Bayes factor is quite difficult because it involves integrating the full dimensional probability distribution function over all model parameters. As mentioned above in our case the parameter space 
can be up to 28 dimensions or even higher. We have made us of the parallel chains that we have run in the characterization phase to compute the evidence via a thermodynamic integration scheme (see e.g. Littenberg \& Cornish (2009) and references therein). After we have computed the evidence for a model with and without a GW signal, we can then construct the ratio of the GW model to the non-GW model, this is known as the Bayes factor. In many cases a Bayes factor larger than 100 is considered decisive evidence and we will adopt that convention here. Figure 3 shows the log of the Bayes factor as a function of the injected signal-to-noise ratio (SNR) for the same noise realization.

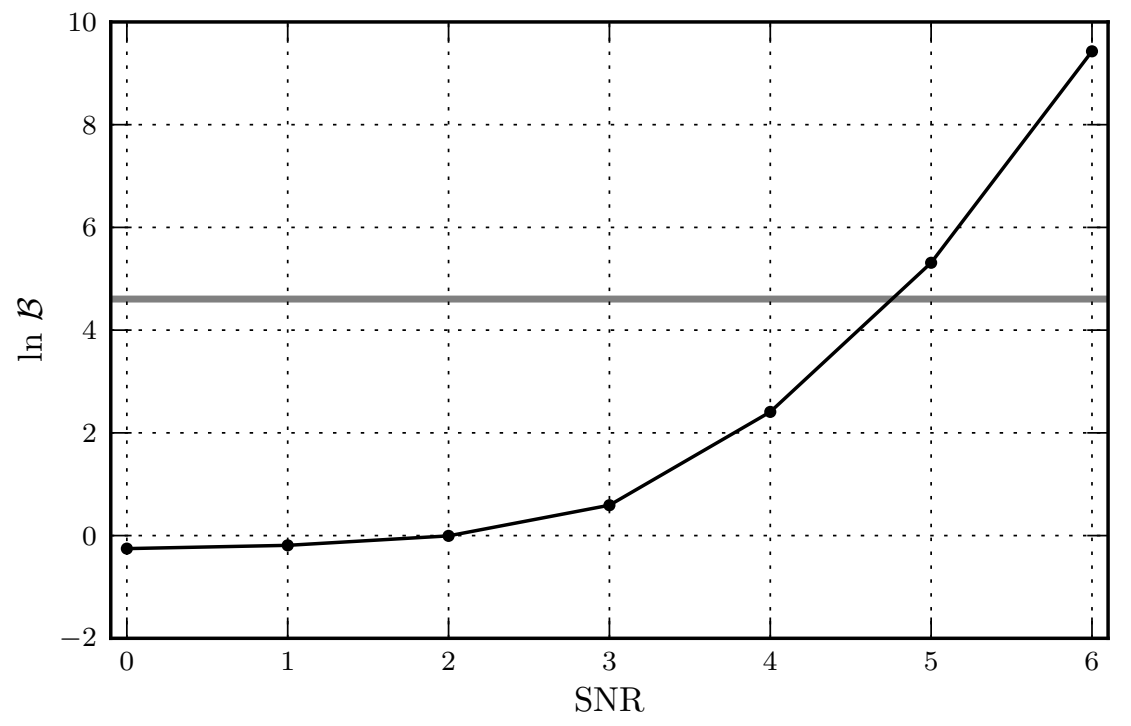

Figure 3: Log of the Bayes factor plotted against injected SNR for the same signal and noise realization. The green horizontal line is the threshold in the log of the Bayes factor in which we can claim a detection and the blue points are the log Bayes factor calculated from thermodynamic integration.

Here we see that the log Bayes factor increases with injected SNR as expected and that a detection is claimed around the SNR $\sim 5$ mark. However, note that this curve can change dramatically based on noise realization.

\section{Conclusion}

In this document, we have reviewed recent progress in the development of a pipeline for detection and characterization of continuous GW sources in PTA data. This algorithm quickly locates the global maximum in parameter space in the search phase, characterizes the GW parameters in the characterization phase, and evaluates the evidence and Bayes factor in the final evaluation stage. In the future we plan on optimizing this algorithm in order to get the quickest possible run time. We also plan to include for the possibility 
of multiple continuous GW source as opposed to the current default of assuming only one source.

\section{References}

Caprini, C., Durrer, R., \& Siemens, X. 2010, D82, 063511

Corbin, V., \& Cornish, N. J. 2010, arXiv:1008.1782

Ellis, J. 2013, ArXiv e-prints

Foster, R. S., \& Backer, D. C. 1990, 361, 300

Hobbs, G., et al. 2010, Classical and Quantum Gravity, 27, 084013

Janssen, G. H., Stappers, B. W., Kramer, M., Purver, M., Jessner, A., \& Cognard, I. 2008, in American Institute of Physics Conference Series, Vol. 983, 40 Years of Pulsars: Millisecond Pulsars, Magnetars and More, ed. C. Bassa, Z. Wang, A. Cumming, \& V. M. Kaspi, 633635

Jenet, F., et al. 2009, arXiv:0909.1058

Lee, K. J., Wex, N., Kramer, M., Stappers, B. W., Bassa, C. G., Janssen, G. H., Karuppusamy, R., \& Smits, R. 2011, 414, 3251

Littenberg, T. B., \& Cornish, N. J. 2009, 80, 063007

Manchester, R. N. 2008, in American Institute of Physics Conference Series, Vol. 983, 40 Years of Pulsars: Millisecond Pulsars, Magnetars and More, ed. C. Bassa, Z. Wang, A. Cumming, \& V. M. Kaspi, 584-592

Olmez, S., Mandic, V., \& Siemens, X. 2010, Phys.Rev., D81, 104028

Romani, R. 1989, in NATO ASI Series, Series C, Vol. 262, Timing Neutron Stars, ed. H. Ögelman \& E. van den Heuvel (Dordrecht, Netherlands; Boston, U.S.A.: Kluwer), $113-117$

Sesana, A., \& Vecchio, A. 2010, 81, 104008

Sesana, A., Vecchio, A., \& Colacino, C. N. 2008, 390, 192

Sesana, A., Vecchio, A., \& Volonteri, M. 2009, 394, 2255

Starobinsky, A. A. 1979, JETP Lett., 30, 682 
Verbiest, J. P. W., Weisberg, J. M., Chael, A. A., Lee, K. J., \& Lorimer, D. R. 2012, 755, 39

Wyithe, J. S. B., \& Loeb, A. 2003, 590, 691 